\title{
AOA-BASED MODELLING AND SIMULATION OF CONSTRUCTION OPERATIONS
}

\author{
Zaneldin, E. K. \\ Department of Civil and Environmental Engineering, United Arab Emirates University, \\ P.O. Box 17555, Al Ain, United Arab Emirates \\ E-mail: essamz@uaeu.ac.ae
}

\begin{abstract}
The construction of building projects is often challenged by tight budgets and limited time and resources. These challenges have forced many construction firms to downsize and re-engineer their operations to improve productivity and cost effectiveness. Re-engineering, however, requires thorough analysis of company-specific operations and resource use, which differ from one construction firm to the other. Simulation can be an effective way of analyzing the operations of construction firms. Developing simulation models, however, is not a simple task and can be complex, time consuming, and costly. In an effort to simplify the modelling process, this paper presents an activity-on-arrow (AOA)-based approach that offers the diversity and flexibility required for construction. The main advantage of this approach is that it uses objectoriented simulation capabilities on traditional AOA networks used for scheduling construction projects. It helps generating practical models without prior knowledge of simulation terminology or the underlying theoretical aspects.

(Received in May 2005, accepted in October 2005. This paper was with the author 1 month for 2 revisions.)
\end{abstract}

Key Words: Computer Applications, Construction, Process Analysis, Modelling, Simulation

\section{INTRODUCTION}

Since the introduction of the CYCLONE system for construction modelling and simulation [1], research in this domain has been growing increasingly [2]. Over the years, several other systems have been developed based on the CYCLONE such as INSIGHT [3], HSM [4], and STROBOSCOPE [5]. Other general-purpose tools have also been introduced for application in construction such as SLAM II [6]. Until recently, however, the software available for simulation has been too complex, too limited, or too costly [7, 8]. The complexities involved in constructing a model and the resulting time requirements have contributed to the limited use of simulation in the construction industry [9]. In addition, the process of developing a simulation model typically requires the user to be familiar with specific terminology and the modelling schematics of a particular software, in addition to the ability to write proprietary computer code. This may not be suitable for many construction practitioners who are not familiar with the operational details needed for accurate simulation. These problems have also contributed to the limited success of simulation in construction.

Several researchers have, therefore, employed different ways to simplify the modelling process and to make it more attractive to practitioners. These include efforts to introduce simulation techniques imported from other domains [10] and other efforts to enhance the operational characteristics of currently used tools (e.g., [9]). The work by Wakefield and Sears [10] applied PETRI Nets, a tool originally used for modelling dynamic systems, to some construction examples to demonstrate their simpler graphical representation and powerful capabilities. They, however, identified the need to use the new terminology of modelling as one of its disadvantages and outlined several potential enhancements to make it more suitable for construction applications. Shi and AbouRizk [9] also proposed a resource-based modelling 
approach with good user-interface and resource libraries to generate a model in SLAM II simulation language. Other research demonstrated how artificial neural networks [11] could be integrated within the modelling environment. Recently, Hajjar and AbouRizk [12] developed a tool that uses an object-oriented environment for construction simulation. Lu [13] presented a discrete-event simulation approach for simulating construction systems. These efforts have provided many interesting features; however, their application is made for simple operations and still requires some familiarity with simulation theory and model representation.

This paper presents a simplified and efficient AOA approach for modelling and simulation of construction operations that is easy to use and does not require prior knowledge of simulation theory. The approach encompasses the AOA representation and powerful process analysis and offers the diversity and flexibility required for construction models. Basic arrow and node objects are first described and their characteristics designed to allow for diverse modelling capabilities. Two example applications are presented. The first demonstrates the ease of modelling of the proposed approach and compares its results to those produced by the CYCLONE system. The second example illustrates the efficiency and practicality of this approach in modelling complex real-world construction operations, and compares its results to those of PETRI Nets.

\section{DEVELOPMENT OF SIMULATION MODELS}

The proposed approach utilizes the object-oriented features to simplify construction modelling and simulation. It simplifies modelling by using the familiar AOA representation of any process, similar to the one used for traditional critical path method (CPM) analysis. The approach also simplifies the simulation process by hiding all queuing and simulation theories to enable the user to focus solely on the logical flow of resources and physical quantities within the process being modelled. The modelling process, as such, becomes intuitive and legible, in addition to being easily performed by a larger base of practitioners.

\subsection{Basic objects}

In the proposed approach, building a simulation model requires two types of drawing objects: arrows and nodes. Using instances of these objects, it is possible to draw a network diagram of any process. Using an AOA representation, the arrows are the activities within the process while the nodes are the control points in the process (see Fig. 1a). The activities consume resources such as labour, equipment, time, and cost. The control nodes, on the other hand, define the flow mechanism to the various branches in the process. The essential challenge in this approach is to set generic properties to the arrow and node objects that allow for the monitoring of the movement/consumption of resources and the flow of quantities produced by resources.

The main properties of an activity (Fig. 1b) define its input requirements and the outputs to be generated at the end of its activation. The input requirements of an activity include: (1) resources and durations; and (2) countable objects. Resources (e.g. loader, truck or construction crew) and their durations are needed to define the cost and time associated with the activity when it is activated. Countable objects, on the other hand, are utilized to simulate any physical quantities that flow through the process (e.g., cubic meters of moved earth, number of piles driven, etc). They are user-defined objects that flow throughout the model from one activity to the other and they maintain the process logic by being specified as generated outputs of predecessors and as input requirements to successors. The generated outputs of an activity include any countable objects that are generated at its end node, which become inputs to succeeding activities. 
The main properties of a node object are designed to control how the succeeding activities are activated during a simulation run. This includes:

- Path Routing: controls the manner by which alternative branches are activated. In Fig. 2a, for example, Node 5 is set with (Path routing = "Probability") so that trucks can have a $5 \%$ chance of breakdown;

- Maximum Queue size: the maximum number of objects permitted to queue before an activity, e.g., the maximum number of trucks that can park at node 5 of Fig. 2a;

- Maximum Copies: e.g., the maximum number of trucks that can simultaneously proceed from node 5 in Fig. 2a; and

- Node Priority: the priority of assigning resources to succeeding activities when these resources are limited in the network (i.e., resources will be given to activities that have higher priorities in the network).

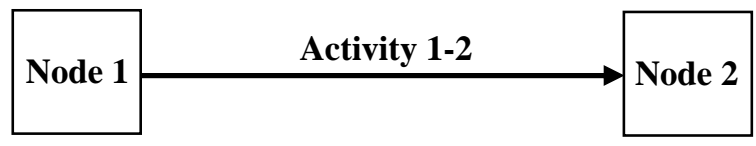

(a) Nodes and Activities

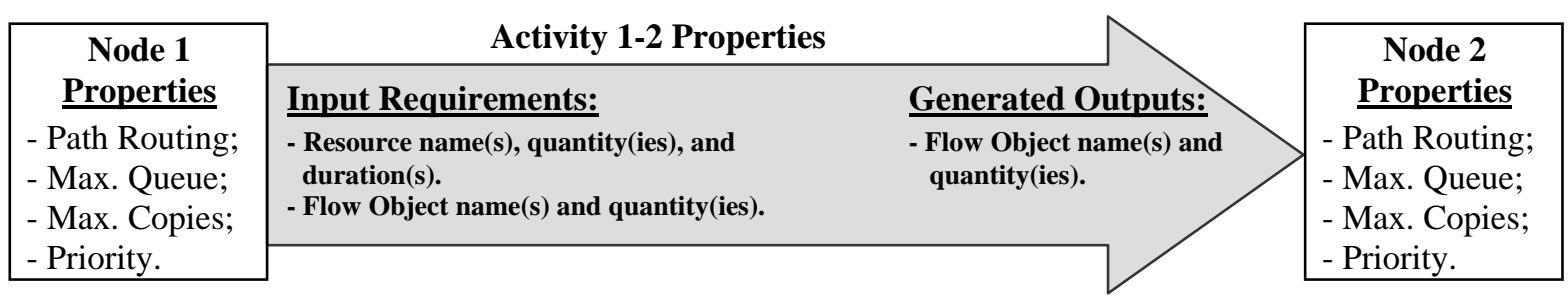

(b) Properties of Nodes and Activities

Figure 1: Basic modelling objects.

\subsection{Simulation mechanism}

Performing a simulation using this approach is simple and does not require prior knowledge of simulation theories. Once a network of a construction process is drawn, the user defines a list of available resources and their hourly rates in a spreadsheet. Next, the user sets the properties of the nodes and activities, including the resources and their durations, and the objects needed and generated for each activity. The simulation process, in essence, is to monitor the movement of resources and countable objects through the model and later analyze the total objects received at specific nodes and the amount of active and idle times.

To model the cyclic nature of a process, the user sets the rate at which new countable objects are generated at the starting node of the model. This is, for example, to model the arrival of a new truck every three minutes (or according to a known distribution) or new concrete batches to be poured. Accordingly, the simulation can be run for a user-specified period of time, starting from the first node and its initial countable object(s). These objects, in addition to existing resources, meet the input requirements of the first activity and accordingly it starts. At the end of its activation, it generates the countable object(s) required for its successor(s). The successor activity(ies) are then activated and the simulation process continues in the same manner, with resources and objects moving according to the logic of the model. When an activity is activated, its start node counts the number of received objects. Also, its end node counts the number of generated objects. 


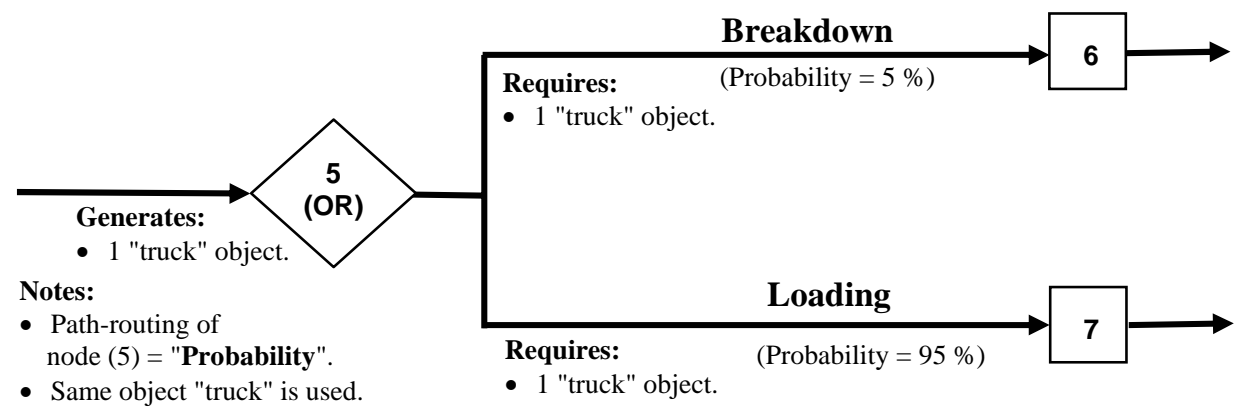

(a) (OR) Connection

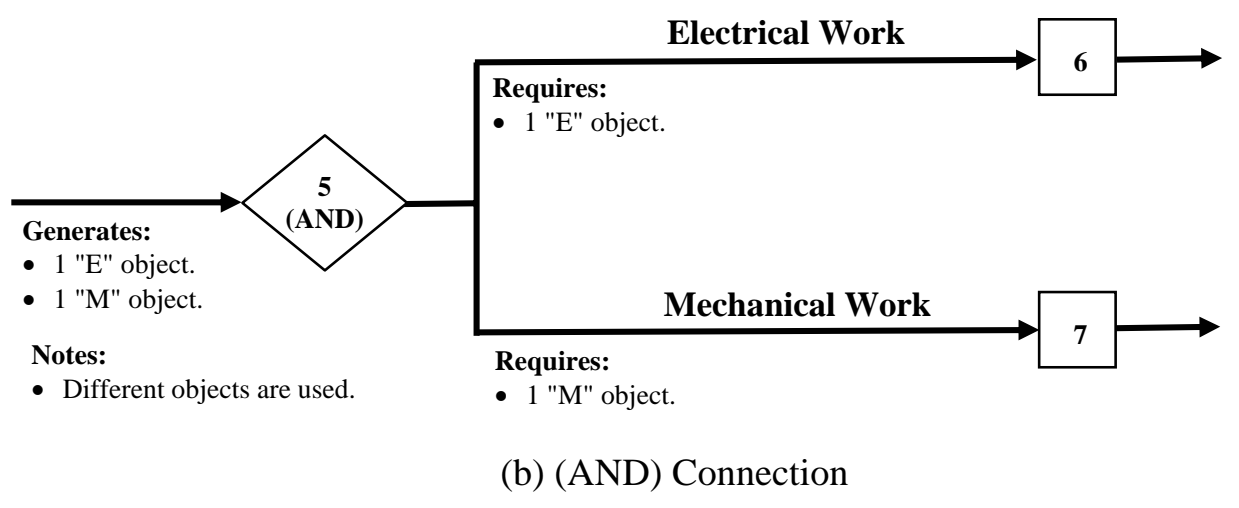

Figure 2: Simple branching.

While it is possible to implement this approach using a variety of means, one elegant implementation has been performed using customizable software for general-purpose simulation [14]. The software, however, uses a proprietary terminology (activities are called work-paths and nodes are called activities) and, due to the general nature, does not provide the diversity needed for developing realistic construction models (advanced objects for conditional branching not included). The software's basic objects therefore are adapted to satisfy the requirements of construction models, as described in the next section.

\section{ADVANCED OBJECTS AND SIMULATION STRATEGIES}

Since construction operations (e.g., earthmoving) exhibit complex environments with multiple resources and changing situations, three improvements to the basic objects have been performed: (1) simple branching objects; (2) conditional branching objects; and (3) advanced process control strategies. Incorporating these improvements in Process v4 software [14] or any other implementation media provides powerful, yet easy-to-use, blocks for building simulation models that suit the construction domain. With little effort, construction managers can simulate high-risk and costly operations before actual construction, and can perform what-if analysis to optimize construction operations.

\subsection{Simple Branching}

Simple branching in the form of (OR) and (AND) nodes is needed to model common construction processes as shown in Fig. 2. An (OR) node is often used to model the branching among mutually exclusive activities by specifying the probability of each activity being activated, as shown in Fig. 2(a). In this example, one "truck" object is generated from the activity prior to the (OR) connection. The same "truck" object is also set as input requirement to each of 
the two activities (5-6) and (5-7). During a simulation run, a "truck" object that is received at node (5) will be routed to either activity, according to the set probability. For example, if 100 objects pass through this node, about 95 objects will go to activity (5-7) and only 5 will go to activity (5-6), considering the given probabilities in Fig. 2(a).

An (AND) connection is needed to model the activation of two parallel paths simultaneously as shown in Fig. 2(b). This type of processing is not readily available in the default options of Process v4 (2003), and as such, a node object had to be designed for it. For the example, parallel activation of activities (5-6) and (5-7) is needed to enable the simultaneous execution of the electrical and mechanical work. The activity prior to the (AND) node is set to generate two different countable objects "E" (required by activity 5-6) and "M" (required by activity 5-7). During a simulation run, the object requirements of activities (5-6) and (5-7) will be available at node (5) and accordingly the activities will be activated simultaneously, providing that their required resources are also available.

\subsection{Conditional branching}

Building upon the simple (OR) and (AND) branching nodes, it is possible to setup realistic model components that cover situations involving conditional branching. Fig. 3(a) and (b) shows two cases where branching is conditional upon the accumulation of a predetermined number of countable objects (e.g., 20 "pile-driven" objects) so that the successor activity (e.g., report to the client) can be started. Fig. 3(c), on the other hand, illustrates a situation in which an activity is conditional upon availability of resources (e.g., aggregates).

The example in Fig. 3(a) represents an (AND-IF) node, which is utilized when an activity can be activated parallel to another activity if a predetermined number of objects are available. It models part of a pile driving operation in which piles are to be filled with concrete once they are driven. Upon driving 20 piles, a progress report is to be sent to the client while continuing with the concreting operation. Similar to a basic (AND) node, the pile driving operation (activity 4-5) generates two different countable objects: one "Pile-ready" and one "Pile-driven". The first object "Pile-ready" is required by activity (5-6) for the concreting operation. Also, 20 of the second object "Pile-driven" are required by activity (5-7) so that the activity can start in parallel with activity 5-6. Node 5 counts the number of objects received and, therefore, postpones the activation of activity (5-7) until twenty "Pile-driven" objects are received.

The example in Fig. 3(b) illustrates an (OR-IF) connection, which is used when only one activity can be activated, conditional upon the availability of countable objects. If this condition is met, only one of the branching activities is activated while all others are deprived from being activated. The example models part of an on-site concreting process. In this operation, concreting is stopped every fifth batch delivered to the site and quality control samples are taken, and after that, concreting continues. The path-routing option of node (4) is set to "priority", with activity (4-5) given a higher priority than activity (4-6). During the simulation, the first delivered concrete batch brings one "Pour" and one "Test" objects at node 4. The "Pour" object flows directly into the concreting activity while the "Test" object waits. The same happens for batches number 2, 3, and 4 . When the fifth batch of concrete is delivered, node 4 will have one "Pour" object and a total of five "Test" objects. These objects are sufficient to activate either activity 4-5 or activity 4-6, but not the two of them. But since activity 4-5 has higher priority, all objects proceed to activity 4-5. The sampling operation (activity 4-5), therefore, starts and activity 4-6 stops. This ensures that concrete pouring stops when samples are being taken. The same process repeats every fifth batch. 


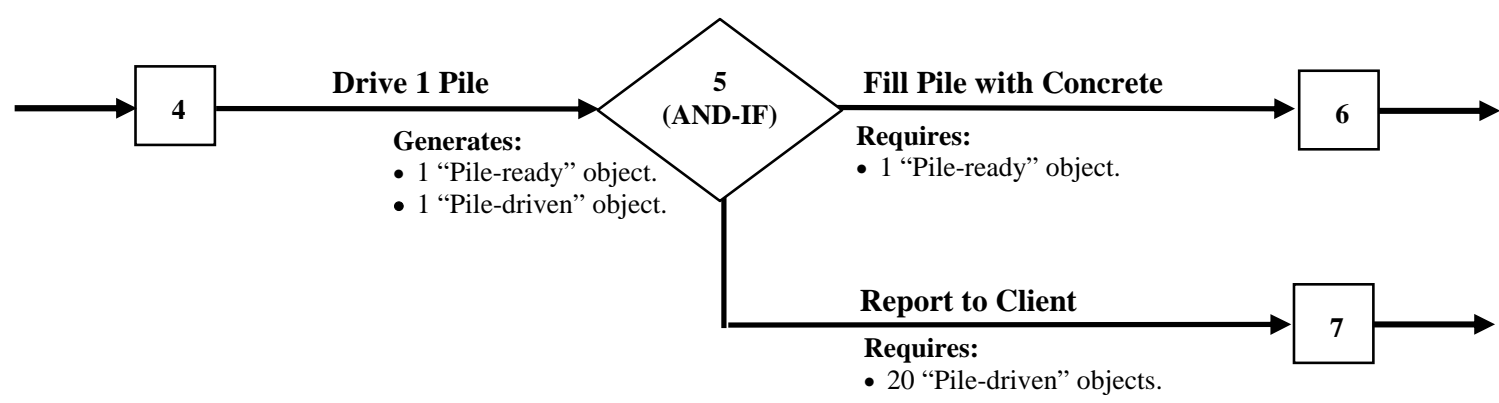

(a) (AND-IF) Node

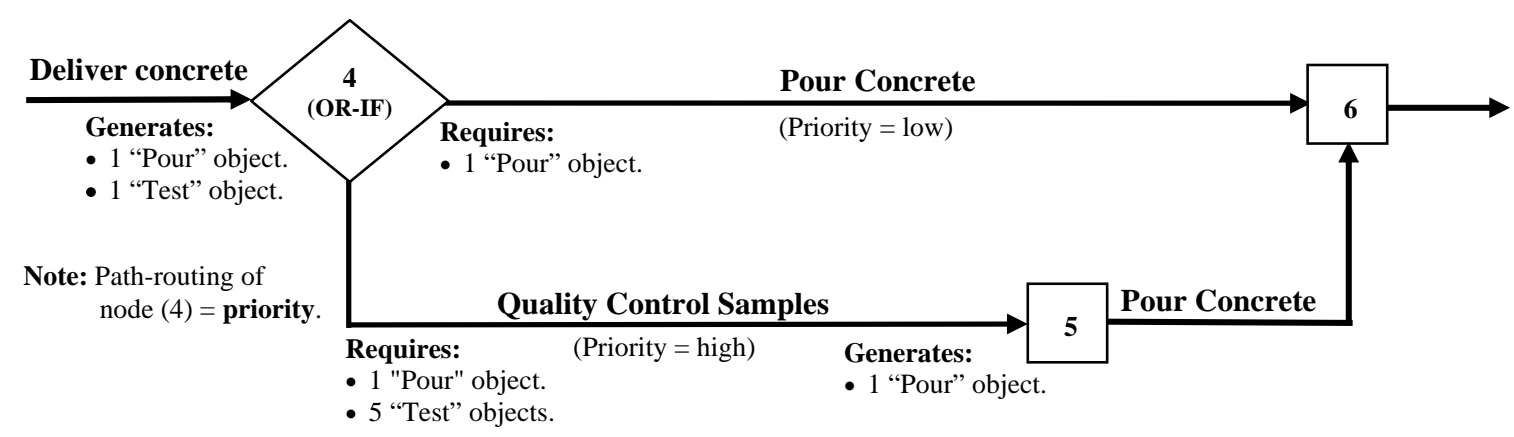

(b) (OR-IF) Node

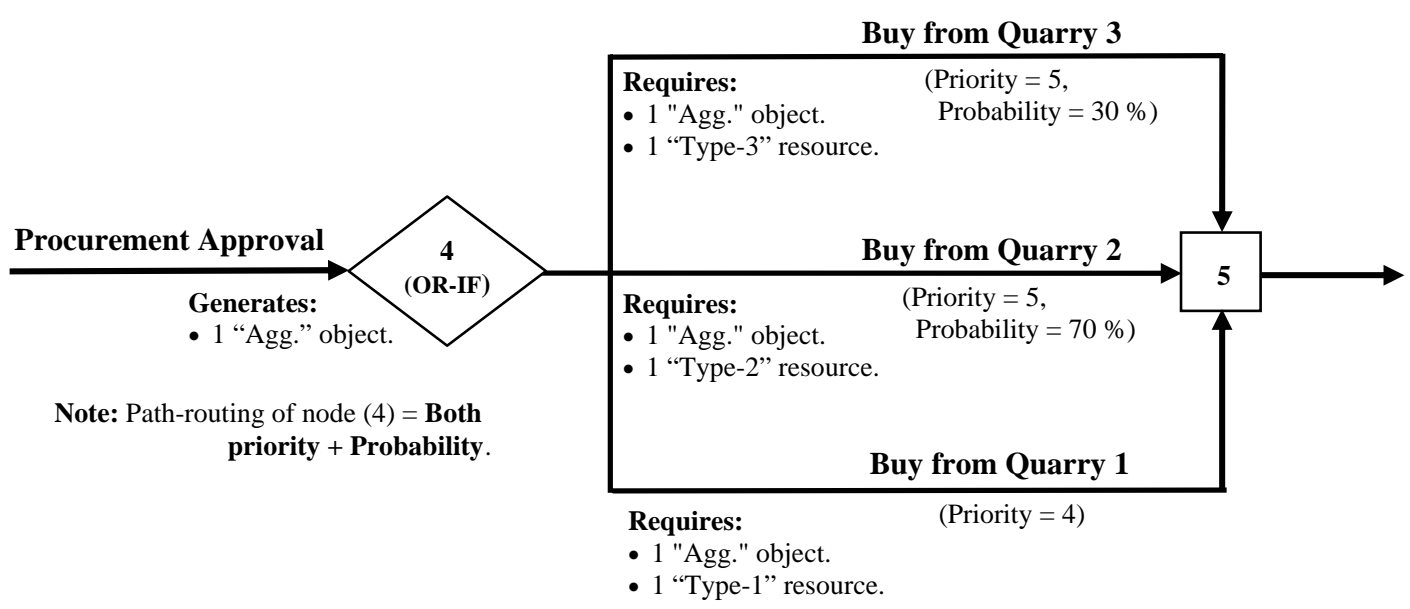

(c) (OR-IF) Node Conditional upon Resources

Figure 3: Conditional branching.

The example in Fig. 3(c) shows another (OR-IF) connection in which processing is conditional upon availability of resources, rather than countable objects. The example models a situation that can be described as follows: "Concrete aggregate will be bought from quarry 1 if aggregate is available, otherwise, aggregate will be bought from either quarry 2 or quarry 3 with probabilities $70 \%$ and $30 \%$, respectively". Objects and resources are assigned to the different activities. The "Agg." object is used in a manner similar to a typical (OR) connection. The main difference in this situation is that the path-routing of node (4) is set to "both", meaning a combination of priority and probability. The "Buy from Quarry 1" activity (the preferred option) is given a higher priority than the other two branching activities. In addition to their low priority, the two activities "Buy from Quarry 2" and "Buy from Quarry 3" are assigned probabilities of 
$70 \%$ and $30 \%$, respectively. This ensures that "Buy from Quarry 1" activity will always be activated if "Type-1" resource is available (activity has higher priority). Otherwise, the other two activities ("Buy from Quarry 2" and "Buy from Quarry 3") will be activated according to their probabilities and the availability of "Type-2" and "Type-3" resources, respectively.

\subsection{Advanced Process Control}

As mentioned previously, the user can set the rate by which new objects enter the simulation. This is, for example, to model the arrival of new trucks to be loaded. In large models, therefore, the new objects make some activities at the beginning of the network to be ready for activation. At the same time, existing objects that are ahead in the model make other activities to be ready for activation. In the event that more than one ready-to-start activity require the same limited resource, a conflict arises and a decision is required on the activity that gets the resource first. The use of node priority (rather than activity priority) in this situation is important to control the assignment of resources in the model. This is also important as it provides alternative strategies for modelling construction operations. The user may, for example, choose to finish existing jobs before starting new ones. This can be modelled by assigning higher priorities to later nodes in the model. Changing this option and re-conducting the simulation provides the project manager with when-if assessment of changing his operational strategy.

\section{APPLICATION EXAMPLES}

Two application examples are analyzed in order to illustrate the use of the proposed object oriented approach for construction modelling and simulation, validate its results, and demonstrate its capabilities. The first example demonstrates the simplicity and modelling ease of the proposed approach, and the second illustrates its efficiency and practicality in modelling complex real-world construction operations.

\subsection{Example 1}

The first example application analyses a simple concrete-placing operation that was originally introduced and modelled by Paulson [15] using INSIGHT, an advanced variation of the CYCLONE system. The process involved placing a number of concrete columns, $2 \mathrm{yd}^{3}$ each, for a new structure. One crane-bucket combination with a capacity of $1 \mathrm{yd}^{3}$ and a flexible "elephant-trunk" was assumed for concrete placement. Concrete was delivered by four trucks, each with a capacity of $8 \mathrm{yd}^{3}$. Because of site constraints, however, only one truck could be moved into the delivery position at a time. One crew of construction workers was also utilized for placing concrete. If a truck and the crane-bucket are both available, then the crane can load the $1-\mathrm{yd}^{3}$ bucket and hoist it to column placement location. The construction crew then uses the bucket to place concrete into a column. The crane and bucket then return for another load. After two buckets are placed, the column is complete and the crew can move to the next column. After the movement of the crew, placement in the new column can begin. It is assumed also that after a truck is emptied, the truck departs and a new truck can enter into the delivery stall.

The proposed approach was effectively applied to develop a simulation model for this concrete placing operation. The model is represented by 4 nodes and 6 arrows (Fig. 4) as opposed to 13 nodes and 18 arrows used to model the same example using INSIGHT (Fig. 5). Notice the activity-on-node representation of traditional simulation tools, as shown in the original INSIGHT model [15]. 


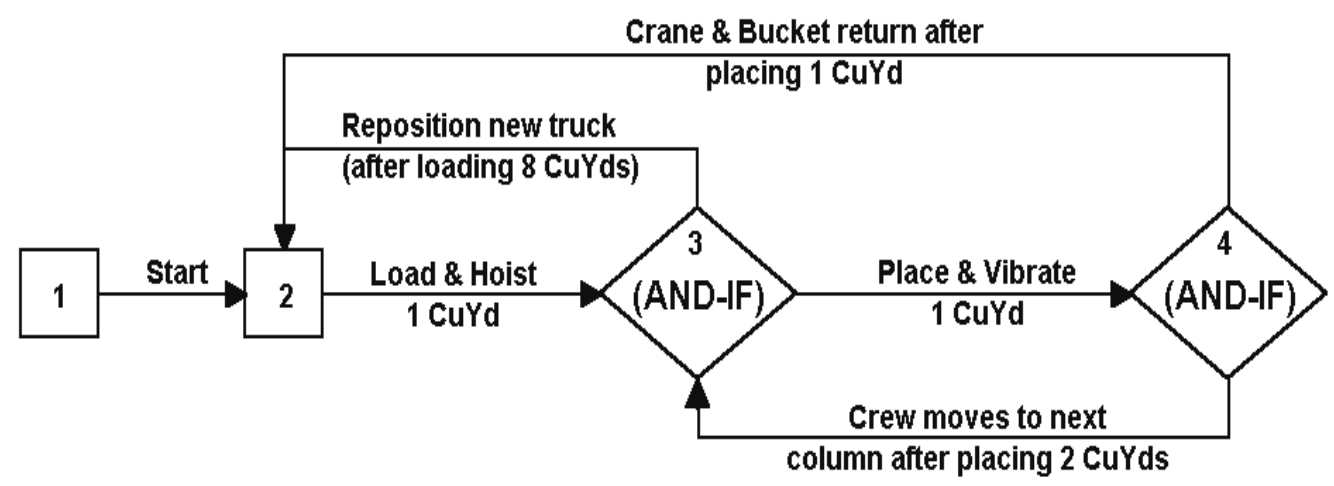

Figure 4: AOA model of Example 1.

For this example, node (2) is a basic node that receives one countable object "cubic yard" from activity 1-2 (start). The successor activity 2-3 (load and hoist 1 cubic yard) requires the availability of one "cubic yard" object and two resources (truck and crane-bucket), and generates two objects "cubic yard" and "truck counter". The following node (3) is an (ANDIF) node that receives the two objects "cubic-yard" and "truck-counter". The first object "cubic-yard" is used to represent the loading of $1 \mathrm{yd}^{3}$ required for activity 3-4 (place and vibrate), and also to count cubic yard production. The second object "truck-counter" represents $1 / 8$ of a full truckload and thus 8 of these objects need to be processed before activity 3-2 (reposition new truck) can be started. Similarly, node (4) is an (AND-IF) node that receives two objects "cubic-yard" and "column-counter" that are generated by activity 34 (place and vibrate). The first object "cubic yard" is required for activity 4-2 (return crane and bucket) while two of the second object "column-counter" are required for activity 4-3 (crew moves to next column).

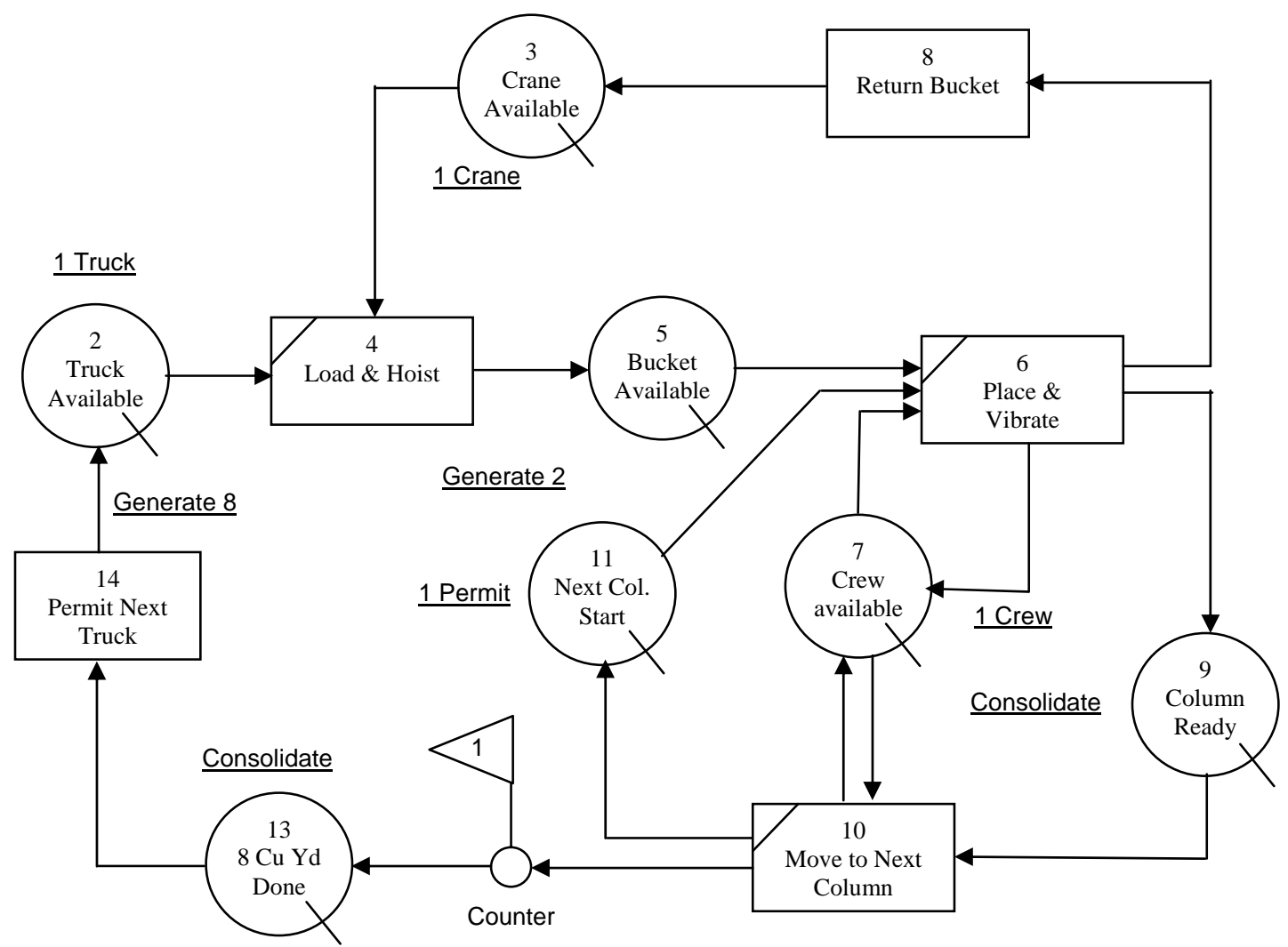

Figure 5: INSIGHT model of Example 1 [15]. 
The cyclic nature of the concrete placing operation is controlled by the number of objects generated at the start node. Since the operation is essentially a sequential one, only one object "cubic-yard" is allowed to enter the simulation through the start node and then rotated over and over through the simulation, following the process network. Using this strategy, the nodes and activities of the model were configured and all nodes were set with copies $=1$, meaning that no activity is allowed to have multiple simultaneous activation (e.g., concreting is done for one column at a time).

From a user's perspective, once the network was drawn, model setup involves first defining the various resources in the resource spreadsheet. The user, then, needs to specify the type and properties of each node and activity. Once done, the process simulation can be started. The user has the option to step through the simulation one-step at a time or to completely run the simulation for a certain period of simulation time (e.g., 1 day). When the first option is used, activated nodes are highlighted and activities with a different colour and provides details on the movement of objects and resources. Following this process, the user will be able to audittrail the simulation and identify any necessary corrections to the model.

In order to enable a comparison with the solution produced by INSIGHT, the present example was run to simulate a full day (8 Hours) of operation. After the simulation was completed, several statistics data became available, which depict the analysis of the cost, time, and resource utilization. Among the useful outputs are activities' total effort, total cost, active time, idle time, interrupted time, maximum queue, average wait, and maximum wait. In terms of simulation results, the total number of activations made by node 4 in activity 4-3 (51 times of crew movements to a new column) and activity 4-2 (103 times of crane and bucket return after placing $1 \mathrm{yd}^{3}$ ). These results provide an hourly production of 6.4 units, very close to the 6.8-unit solution provided by Paulson [15], given the probabilistic nature of the model.

As such, the results demonstrate the validity of the proposed approach and indicate that it is capable of generating results that are in close agreement with those produced by INSIGHT system. In addition, the proposed approach provides an added advantage of simplicity and ease of use. The approach provides a model representation for this example that is much simpler, easier to model, and more legible than that provided by INSIGHT. The developed model includes only 4 nodes and 6 arrows as opposed to 13 the nodes and 18 arrows used in the INSIGHT model. As such, the application of the proposed approach significantly contributes to simplifying and reducing the complexity of modelling as it was proven to reduce the number of needed nodes and arrows by $69 \%$ and $67 \%$, respectively, for this example.

\subsection{Example 2}

This example is analyzed to illustrate the capabilities of the proposed approach in simulating real-world and complex construction earthmoving operations. The example analyses the earthmoving operation at the New Hong Kong International Airport site that was previously modelled by Wakefield and Sears [10] using PETRI Nets. The construction operation involved loading excavated material into trucks using a one Demag H285 loader. Based on the quality of the excavated material, it was hauled into one of three fill locations (Site A, Site B, or Site C). Two types of trucks were used on site: (1) CAT777 trucks with capacity 30, 33, or $35 \mathrm{~m}^{3}$ for hauling material type A, B, or C, respectively; and (2) CAT785 trucks with capacity 50, 55, or $60 \mathrm{~m}^{3}$ for hauling material type A, B, or C, respectively. In addition, three alternative truck-fleet combinations were available as options in the operation and thus were compared in different simulation experiments: (1) five CAT785 trucks; (2) eight CAT777 trucks, or (3) three CAT785 plus four CAT777 trucks. 
In the operation, trucks are first loaded, one-at-a-time, using the Demag H285 loader. During loading, either material type (A, B, or C) may be encountered, according to a known probability. Consequently, the proper truck capacity, hauling time, and destination site are selected. For practicality, truck breakdown probabilities were also used to model the situation in which one type of truck is more reliable than another. Once the excavated material is loaded, it is then hauled and dumped at the appropriate fill location (Site A, Site B, or Site C). Finally, the trucks return back into the loading positions. The work was carried out in two 11hour shifts per day.

The simulation model of this operation using PETRI Nets (Fig. 6) involved 42 nodes of different types and 56 arrows. Using the proposed approach, model representation (Fig. 7) uses only 16 nodes and 23 activities. As shown in the figure, node 2 is an (AND) node while nodes 3 and 4 are (OR) nodes. Similar to example 1, the input requirements and generated outputs of all activities were used to setup the nodes and activities of the model. Three countable objects (Mat. A, Mat. B, and Mat. C) were used to accumulate the quantity of fill material dumped at each site. Also, higher priorities were given to the later nodes in the model to ensure that the truck resource is assigned to the dumping and return operations, before it is assigned to the loading of a new load. Daily production results of the simulation with the three truck fleet combinations are given in Table I, using the proposed approach and PETRI Nets. The difference in results between the proposed approach and PETRI Nets is expected and can be attributed to the probabilistic nature of the model and the inability to provide a triangular distribution for the loading time in the simulation tool used in this paper.

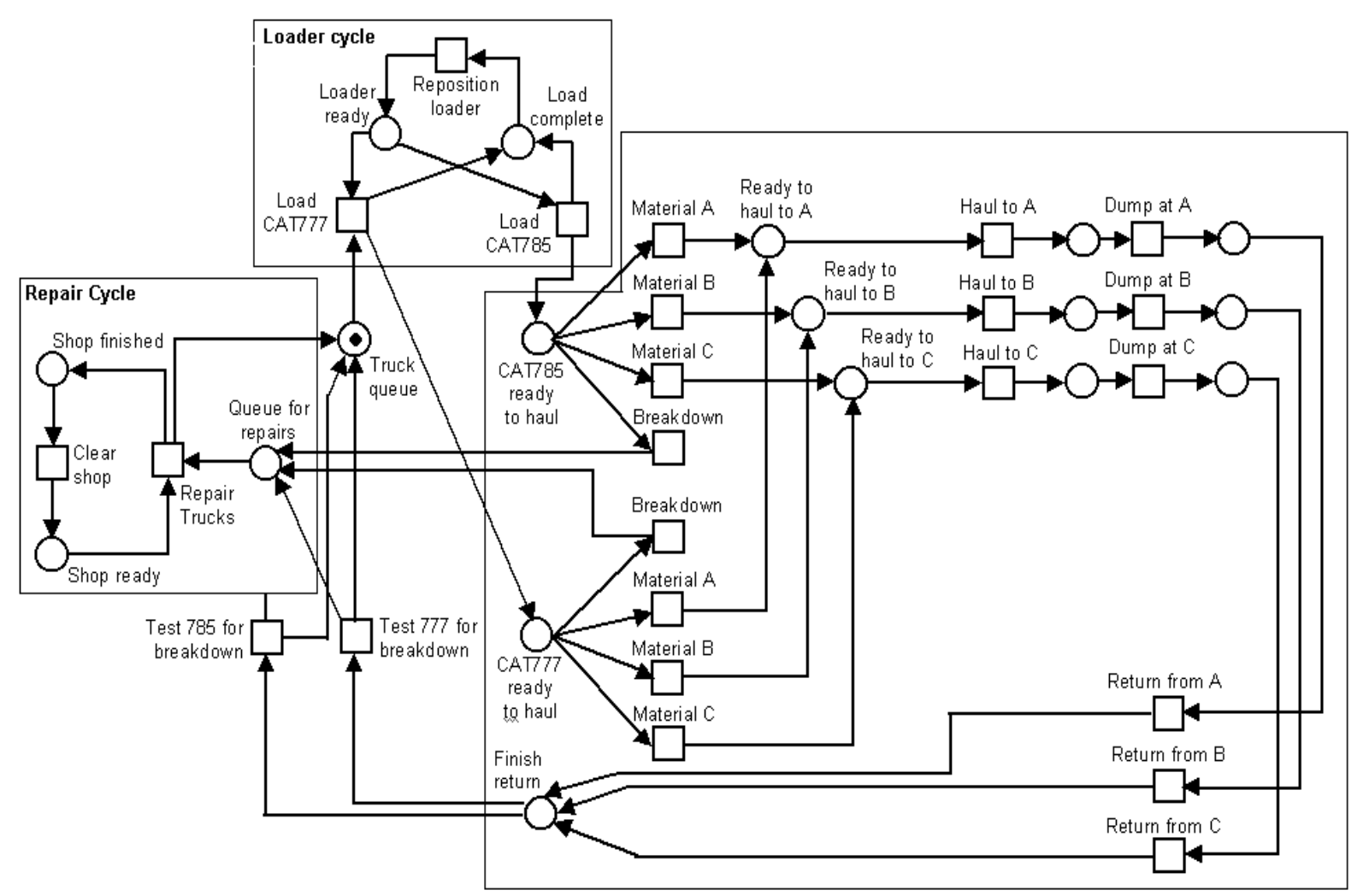

Figure 6: PETRI Nets model of Example 2 [10].

This example illustrates the capabilities of the proposed approach in modelling complex and real-world construction operations that require heterogeneous queues, non-deterministic transitions, different load destinations, and breakdown probabilities. The example also 
demonstrates the simplicity, efficiency and conciseness of the models produced by the present approach. For this example, the application of the present approach was proven to significantly reduce the complexity of the developed simulation model. The model required $62 \%$ and $59 \%$ fewer nodes and arrows, respectively, compared to those used in the PETRI Nets model. In addition, the various reports produced by the utilized simulation software provide valuable information pertaining to resource utilization, production costs, active and idle times that can be used by contractors to determine the optimum earthmoving system.

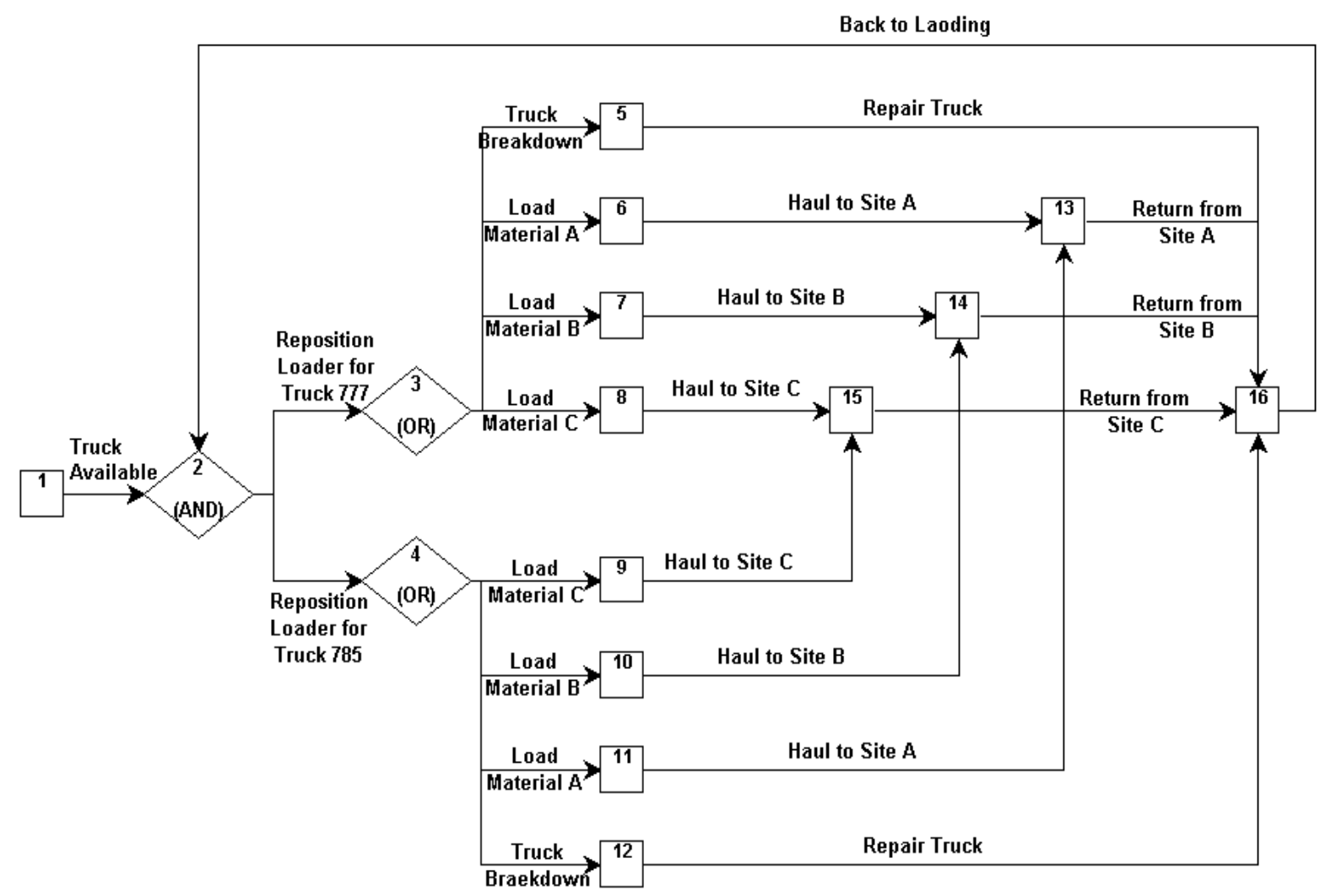

Figure 7: AOA model of Example 2.

Table I: Average daily quantity at the three dump locations of Example 2.

\begin{tabular}{|c|c|c|c|c|c|c|}
\hline \multirow[b]{2}{*}{ Truck Fleet Options } & \multicolumn{2}{|c|}{ Site $\mathbf{A}\left(\mathbf{m}^{3}\right)$} & \multicolumn{2}{|c|}{ Site B $\left(\mathbf{m}^{3}\right)$} & \multicolumn{2}{|c|}{ Site C $\left(\mathrm{m}^{3}\right)$} \\
\hline & $\begin{array}{l}\text { Proposed } \\
\text { Approach }\end{array}$ & $\begin{array}{l}\text { PETRI } \\
\text { Nets }\end{array}$ & $\begin{array}{l}\text { Proposed } \\
\text { Approach }\end{array}$ & $\begin{array}{l}\text { PETRI } \\
\text { Nets }\end{array}$ & $\begin{array}{l}\text { Proposed } \\
\text { Approach }\end{array}$ & $\begin{array}{c}\text { PETRI } \\
\text { Nets }\end{array}$ \\
\hline 5 CAT785 & 8510 & 9161 & 6273 & 6865 & 5580 & 5994 \\
\hline 8 CAT777 & 7033 & 8475 & 5305 & 6406 & 4801 & 5447 \\
\hline 3 CAT785 + 4 CAT777 & 7936 & 7744 & 5771 & 5719 & 5076 & 5025 \\
\hline
\end{tabular}

\section{SUMMARY AND CONCLUDING REMARKS}

It is well established in the literature that computer simulation tools are useful for analyzing construction operations. In an attempt to spur a wide use of such tools within the industry, this paper presented a simple and powerful modelling approach for use in construction. Using the 
modelling components described in this paper, realistic models can be constructed with relative ease and in a legible format. The elegant object-oriented nature of this approach makes it convenient for users who are not familiar with simulation theories and helps them focus more on the accurate mapping of their process rather than on programming and syntax issues. Two example applications were presented to demonstrate the applicability of this approach in construction and to show its comparable results with two approaches used in construction, CYCLONE and PETRI Nets. The two examples illustrated the validity of the proposed approach and its capabilities in modelling complex construction operations. The main advantages of the proposed approach are its simplicity, ease of use, and practicality. The ultimate goal is to reduce the barriers between simulation and the construction industry and make the process of building simulation models even more cost effective.

\section{REFERENCES}

[1] Halpin, D. W. (1973). An investigation of the use of simulation networks for modeling construction operations, $P h D$ thesis, University of Illinois at Urbana-Champaign, USA

[2] Martinez, J. C.; Ioannou, P. G. (1999). General-purpose systems for effective construction simulation, Journal of Construction Engineering and Management, Vol. 125, No. 4, 265-276

[3] Paulson, B. C. Jr. (1978). Interactive graphics for simulating construction operations, Journal of Construction Div., ASCE, Vol. 104, No. 1, 69-76

[4] Sawhney, A.; AbouRizk, S. M. (1995). Simulation based planning method for construction project, Journal of Construction Engineering and Management, Vol. 121, No. 3, 297-303

[5] Martinez, J. C. (1996). STROBOSCOPE: State and resource based simulation of construction processes, Ph.D. Dissertation, University of Michigan, Ann Arbor, USA

[6] Gonzalez-Quevedo, A. A.; AbouRizk, S. M.; Isley, D. T.; Halpin, D. W. (1993). Comparison of two simulation methodologies in construction, Journal of Construction Engineering and Management, Vol. 119, No. 3, 573-589

[7] Paulson, B. C. Jr. (1995). Computer applications in construction, McGraw-Hill, Inc., NY, USA

[8] Marzouk, M.; Moselhi, O. (2003). Object-oriented simulation model for earthmoving operations, Journal of Construction Engineering and Management, Vol. 129, No. 2, 173-181

[9] Shi, J.; AbouRizk, S. M. (1997). Resource-based modeling for construction simulation, Journal of Construction Engineering and Management, Vol. 123, No. 1, 26-33

[10] Wakefield, R. R.; Sears, G. A. (1997). PETRI Nets for simulation and modeling of construction systems, Journal of Construction Engineering and Management, Vol. 123, No. 2, 105-112

[11] Hajjar, D.; AbouRizk, S. M.; Mather, K. (1998). Integrating neural networks with special purpose simulation, Proceedings of the 1998 Winter Simulation Conference, IEEE, 1325-1332

[12] Hajjar, D.; AbouRizk, S. M. (2002). Unified modeling methodology for construction simulation. Journal of Construction Engineering and Management, Vol. 128, No. 2, 174-185

[13] Lu, M. (2003). Simplified Discrete-Event Simulation Approach for Construction Simulation, Journal of Construction Engineering and Management, Vol. 129, No. 5, 537-546

[14] Process v4. (2003). User's Guide, Sciforma Corporation, Los Gatos, CA, USA

[15] Paulson, B. C. Jr. (1987). Construction operations simulation by microcomputer, Journal of Construction Engineering and Management, Vol. 113, No. 2, 302-314 